\title{
The role of nanodefects in gas barrier layers
}

\author{
Vincent Tobin, Ashley Sim, Hélène Suttle, Hazel Assender \\ Department of Materials, University of Oxford, 16 Parks Road, Oxford OX1 3PH \\ hazel.assender@materials.ox.ac.uk, +44 1865283715
}

\begin{abstract}
Barrier performance of continuous coatings is determined by the defect population in the barrier layer. In this paper we consider two classes of defects: those in which the permeant does not interact with the barrier material, 'macrodefects', and those in which there is an interaction between the permeant and the barrier material, 'nanodefects', thus leading to a greater activation energy for permeation than that of permeation through the underlying polymer. If a barrier is improved by reducing the density of macrodefects then typically the transmission rate will decrease, and eventually the activation energy will increase as the proportion of permeation that takes place through the nanodefect population becomes significant. Recent studies, however, have highlighted examples in which a greater activation energy is associated with a greater permeation. This is explored in terms of a simple double-Arrhenius model, which highlights the significant contribution from nanodefect permeation even in cases in which the overall barrier performance is not that high.
\end{abstract}

\section{Introduction}

\section{Gas barrier layers}

Gas barrier films consisting of notionally continuous layers of some low permeation material, such as an oxide, are of interest for encapsulation of highly water-sensitive materials, including those used in flexible electronics. The flexible, usually polymeric, substrate is typically much more permeable to water vapour than the barrier coating, and if the barrier were completely continuous, the permeability of the composite would be extremely low. However, we know that in practice the barrier layers contain defects, various in nature, which allow the permeation of vapour. [1] The origin of such defects may be inherent to the structure of the coating layer e.g, from the network structure of an amorphous solid or very small defects associated with the boundaries between agglomerates of material, formed during nucleation and growth of the layer during vapour deposition [2], from asperities or roughness on the surface of the underlying substrate [3], or, at a larger scale, due to defects induced by arcing during deposition, or handling scratches or cracks [4].

\section{Activation energy of permeation}

The activation energy of permeation can be determined from a series of permeation measurements at different temperatures. The activation energy can be related to the permeation, in the case of water vapour the water vapour transition rate, $W V T R$, by the Arrhenius relation:

$$
W V T R=W V T R_{0} \exp \left(-E_{A} / R T\right)
$$

in which $W V T R_{0}$ is the pre-exponential coefficient, $E_{A}$ is the activation energy, $R$ the molar gas constant and $T$ the absolute temperature.

Measurement of the activation energy of gas permeation has been used as an indicator of the nature of the defects through which the gas permeates. In this paper we define just two classes of defect: 
i) Macrodefects. In this case there is negligible interaction between the permeant and the walls of the defect, and hence the activation energy of a composite material with permeation exclusively through macrodefects in the barrier layer will be equal to the activation energy of the underlying substrate (that of the uncoated polymer).

ii) Nanodefects. In this case there is significant interaction between the permeating species and walls of the defect [1,5-7], and so the activation energy of the composite is something greater than that of the underlying polymer, reflecting the nature of the nanodefect.

The overall measured composite activation energy can therefore be modelled as the sum of the WVTR through the macrodefects and the WVTR of the nanodefects:

$W V T R=W V T R_{m 0} \exp \left(-E_{m A} / R T\right)+W V T R_{n 0} \exp \left(-E_{n A} / R T\right)$

where $W V T R_{m 0}$ and $W V T R_{n 0}$ are the pre-exponential coefficients for macrodefect and nanodefect permeation respectively, and $E_{m A}$ and $E_{n A}$ are the activation energies of macrodefect and nanodefect permeation respectively. This sum does not fit a single exponential for composite permeation such as that in equation (1) over all temperatures, but for the narrow range of temperatures that are measured experimentally, the data do fit a straight line in a plot of In (WVTR) vs. 1/T to a good approximation, and thus an apparent activation energy for the combination of macrodefect and nanodefect permeation can be determined. $E_{m A}$ is equal to the activation energy of the underlying polymer, and can thus be determined from permeation measurements of uncoated polymer.

\section{Macrodefect and Nanodefect populations}

If we consider a barrier layer with only a macrodefect population, then as the density of macrodefects is decreased the activation energy of the composite remains constant, but the overall WVTR decreases as the area of apparently exposed polymer decreases (Figure 1).

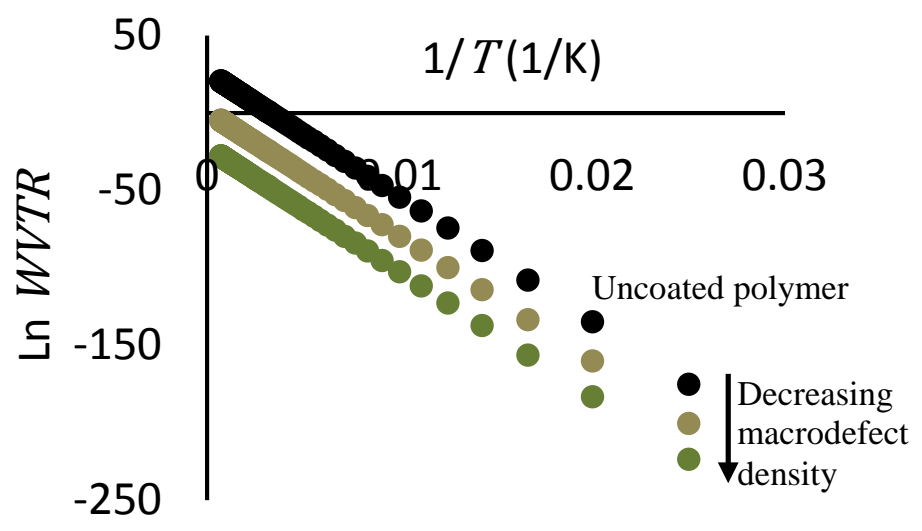

Figure 1: Schematic of WVTR data for uncoated polymer and two composite films with decreasing density of macrodefects, illustrating how the activation energy (the gradient of the graph) remains constant, but the permeation decreases.

For the component of permeation due to nanodefects, the gradient of the graph is greater, and the sum of the macrodefect and nanodefect contributions is illustrated in Figures 2 and 3. 


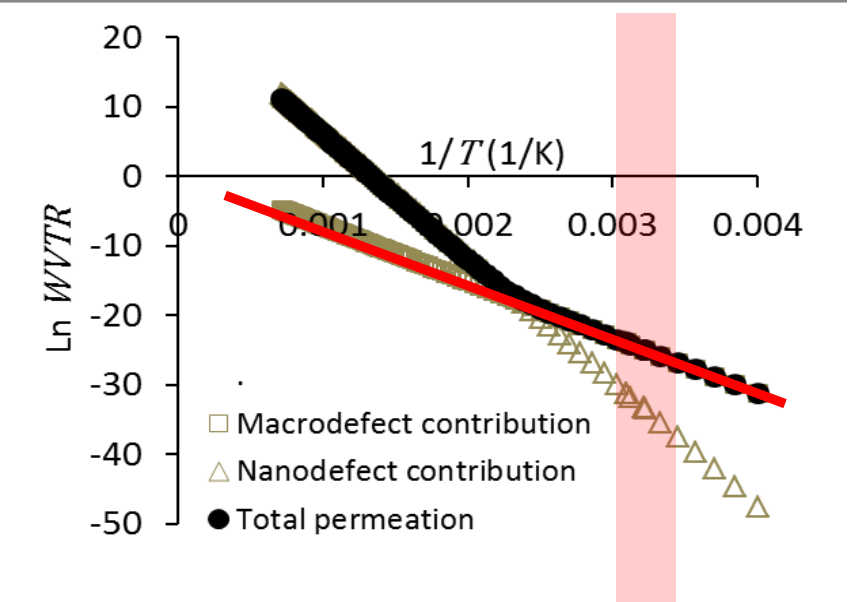

Figure 2: Schematic of WVTR data for a coated polymer with a particular combination of macrodefect and nanodefect density. With the populations in this example, permeation through macrodefects dominates within the experimental range of temperatures (indicated with the shaded box), and the apparent composite activation energy (indicated by the solid line) has a gradient equal to that of the uncoated polymer.

Previous work has often considered the case illustrated in Figure 2, in which permeation is dominated by that through macrodefects, and changing the population of macrodefects improved the permeation, but does not alter the activation energy.

Figure 3 illustrates a case in which, at the experimental range of temperature, the macrodefect permeation is more comparable to the permeation through nanodefects, and in this case the activation energy becomes greater than that of the base polymer. Where the activation energy is seen to rise [8], this has been interpreted as being due to a decrease in macrodefect population to such an extent that nanodefect permeation becomes significant [9]. In this case, as the macrodefect population is reduced, the permeation continues to decrease, and the activation energy starts to increase. A greater activation energy therefore has tended to be associated with a lower permeation.

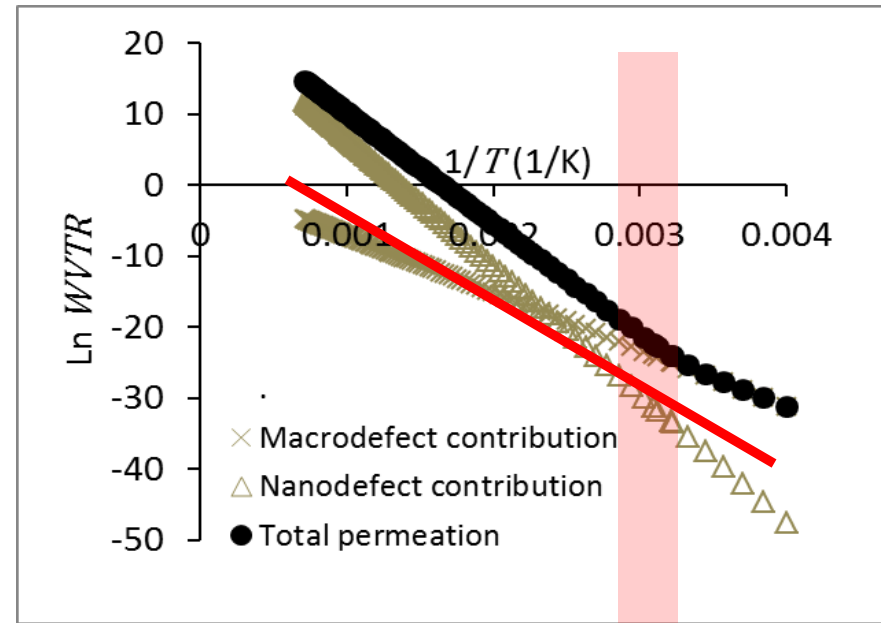

Figure 3: Schematic of WVTR data for a coated polymer with a particular combination of macrodefect and nanodefect density. With the populations in this example, permeation is through a combination of macrodefects and nanodefects within the experimental range of temperatures (indicated with the shaded box), and the apparent composite activation energy (indicated by the solid line) has a gradient greater than that of the uncoated polymer. 
The increase in activation energy, however, only indicates a change in balance between permeation through nanodefects and through macrodefects, and so changes in nanodefect density must also be considered.

\section{Experimental methods}

Two polymer substrates were used for barrier layers: PEN of thickness $125 \mu \mathrm{m}$ tailored to have a very smooth upper surface (Teonex Q65, DuPont Teijin) to receive a barrier coating, and bi-axially oriented polypropylene of thickness 90 $\mathrm{m}$ (Rayoart CG 90, Innovia Films) that does not have such surface conditioning.

Aluminium oxide (AIOx) barrier coatings were deposited by RF magnetron reactive sputtering from Al targets (Angstrom Sciences) in a roll-to-roll vacuum webcoating facility (Aerre Machines). Substrates were taped to a drum (cylinder width $350 \mathrm{~mm}$, circumference $2 \mathrm{~m}$ ) rotating at $25 \mathrm{rpm}$, meaning that during a single deposition run, the sample passes the sputter cathodes multiple times, building up a single, thick, oxide layer made up of multiple sublayers. The target poisoning point was established before each set of samples, and the deposition condition held just before poisoning, with typical gas flows of $15 \mathrm{sccm}$ oxygen and $200 \mathrm{sccm}$ argon.

Surface topography of substrates and coatings was imaged using Ominscan MicroXAM 5000B 3d ADE Phase shift interference contrast optical profiler. Coating thickness was measured using a Rudolph Auto EL ellipsometer on films deposited onto fragments of silicon wafer placed alongside the polymer during deposition.

WVTR was recorded using the MOCON Permatran W $3 / 31$ (ASTM F- 1249 ) at $35^{\circ} \mathrm{C}, 38^{\circ} \mathrm{C}, 44^{\circ} \mathrm{C}$ and $50^{\circ} \mathrm{C} 100 \% \mathrm{RH}$ with a sample size $50 \mathrm{~cm}^{3}$. Ca test measurements were carried out to qualitatively assess the permeation. Four $2 \mathrm{~cm}$ diameter discs of $\mathrm{Ca}$ were evaporated onto the coated side of the barrier films in an Edwards 306 evaporator (2-5 x 10-5 mBar at deposition rate of 0.3-0.6 $\left.\mathrm{nm} \mathrm{s}^{-1}\right)$ located within a nitrogen atmosphere glovebox. $3 \mathrm{~mm}$ thick float glass squares (cleaned with isopropanol, water and then dried in a vacuum oven for at least 3 hours) were adhered to the barrier layer with the Ca layer on the glass side using about 20ml of epoxy (Electrolite Corporation, ELC-2500) cured with a hand-held UV lamp (Intertronics IUV250). In this way, any water vapour that degrades the reflective $\mathrm{Ca}$ to transparent $\mathrm{Ca}(\mathrm{OH})_{2}$ must permeate through the barrier composite. The samples were stored in an oven held at $38^{\circ} \mathrm{C}$ with $85 \% \mathrm{RH}$ atmosphere (equilibrated with a saturated aqueous solution of potassium chloride) and the Ca spots were photographed (Olympus SP-320 camera) at appropriate intervals, the sample lit in transmission.

\section{Results and Discussion}

Several examples have now been observed in which the nanodefect density has been observed to change between sample conditions, and it is this change in nanodefects, rather than macrodefects that has been found to influence the permeation behaviour. Here, we present an example in which the nature of the polymer substrate has influenced the balance of macrodefect and nanodefect permeation, and hence, unexpectedly, we observed a greater activation energy in films that showed a higher degree of permeation.

We compared the permeation behaviour in the temperature range $35^{\circ} \mathrm{C}$ to $50^{\circ} \mathrm{C}$ of $\mathrm{AlOx}$ coatings on Teonex Q65 (DuPont Teijin Films), a PEN substrate of thickness $125 \mu \mathrm{m}$ with a highly smooth surface finish, with that of

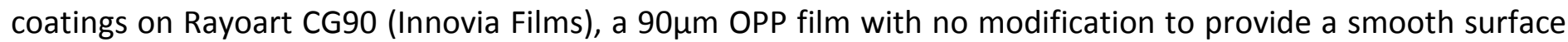
finish. In both cases the AlOx was deposited by reactive RF magnetron sputtering using our roll-to-roll webcoating facility. The samples were attached directly to the coating drum, allowing for multiple passes of the substrate past the coating source to build up an AlOx layer of about 145nm in thickness. Previous work [10-12] has shown that such a multiple pass method allows us to build relatively thick oxide layers without a significant loss in barrier properties as the thickness increases. Table 1 presents permeation data and parameters fitted to equation (2). The permeability, $P$, of the coating is calculated using ideal laminate theory [13] where: 


$$
P=W V T R \times t / \Delta p
$$

with $t$ being the film thickness and $\Delta p$ being the pressure difference across the film, and:

$$
P_{\text {composite }}=\left(\varphi_{\text {polymer }} / P_{\text {polymer }}+\varphi_{\text {coating }} / P_{\text {coating }}\right)^{-1}(4)
$$

where $\phi$ is the volume fraction.

As shown in Table 1, we observe experimentally that the OPP/AIOx film has a greater overall apparent activation energy despite being a poorer barrier to water vapour. The OPP substrate itself is a better barrier to water than PEN, but the performance, as calculated using ideal laminate theory, of the coating alone on the PEN is far superior to that on the OPP.

Table 1: Experimental and equation fitting parameters for permeation through AlOx coated polymer films: a comparison of the permeation through barrier films based on a smooth PEN substrate and a rough OPP

\begin{tabular}{|c|c|c|c|}
\hline & & $\begin{array}{l}\text { OPP/ } \\
\text { AlOx }\end{array}$ & $\begin{array}{l}\text { PEN/ } \\
\text { AlOx }\end{array}$ \\
\hline \multirow{5}{*}{$\begin{array}{l}\text { Experi- } \\
\text { mental }\end{array}$} & Polymer thickness $(\mu \mathrm{m})$ & 90 & 125 \\
\hline & AlOx thickness ( $\mu \mathrm{m})$ & 0.145 & 0.148 \\
\hline & WVTR $38^{\circ} \mathrm{C}\left(\mathrm{g} / \mathrm{m}^{2} /\right.$ day $)$ & 0.38 & 0.054 \\
\hline & $\Delta E(\mathrm{~kJ} / \mathrm{mol})$ & 104 & 84 \\
\hline & Permeability of coating & 0.075 & 0.0083 \\
\hline \multirow{3}{*}{$\begin{array}{l}\text { Macro- } \\
\text { defects }\end{array}$} & $W V T R_{m o}\left(\mathrm{~g} / \mathrm{m}^{2} /\right.$ day) & $3.6 \times 10^{10}$ & $4.3 \times 10^{7}$ \\
\hline & $\Delta E_{m}(\mathrm{~kJ} / \mathrm{mol})$ & 67 & 54 \\
\hline & $W_{V T R} 38^{\circ} \mathrm{C}\left(\mathrm{g} / \mathrm{m}^{2} /\right.$ day $)$ & 0.20 & 0.04 \\
\hline \multirow{3}{*}{$\begin{array}{l}\text { Nano- } \\
\text { defects }\end{array}$} & $W V T R_{n o}\left(\mathrm{~g} / \mathrm{m}^{2} /\right.$ day $)$ & $12.4 \times 10^{20}$ & $0.9 \times 10^{20}$ \\
\hline & $\Delta E_{n}(\mathrm{~kJ} / \mathrm{mol})$ & 130 & 130 \\
\hline & $W V T R_{n} 38^{\circ} \mathrm{C}\left(\mathrm{g} / \mathrm{m}^{2} /\right.$ day $)$ & 0.18 & 0.01 \\
\hline \multirow{3}{*}{ Total } & WVTR $38^{\circ} \mathrm{C}\left(\mathrm{g} / \mathrm{m}^{2} /\right.$ day $)$ & 0.38 & 0.05 \\
\hline & $\Delta E(\mathrm{~kJ} / \mathrm{mol})$ & 104 & 84 \\
\hline & $W V T R_{n} / W V T R$ & $48 \%$ & $27 \%$ \\
\hline
\end{tabular}
substrate.

This difference in barrier performance arises due to the effect of the underlying substrate on the barrier performance of the coating. The difference in chemistry of the polymer substrate may play a part - we would expect a lower nucleation density of oxide on the OPP surface during deposition, in comparison to that on PEN [2], and the adhesion of the coating to the substrate may also play a role: where there is poor adhesion there is the possibility for gas to rapidly permeate along the interface between the substrate and the oxide until it finds a defect in the oxide through which to pass, but the surface roughness of the substrate polymer is likely to have a substantial role to play in the initiation of defects in the subsequently deposited oxide. Figure 4 shows optical profilometry images of the oxide surfaces on each of the polymer substrates. The OPP surface is seen to be considerably rougher, with a high density of features more than a micrometre in height, which is reflected in the oxide surface. By contrast, the PEN shows a very low number of asperities: in this field of view only two that are comparable or greater than the thickness of the oxide layer itself. It might be the case that such asperities are associated with macrodefects, in which case we would expect that permeation due to macrodefects would be greater in the case of the OPP. Similar morphology is seen in the uncoated polymer in each case. 


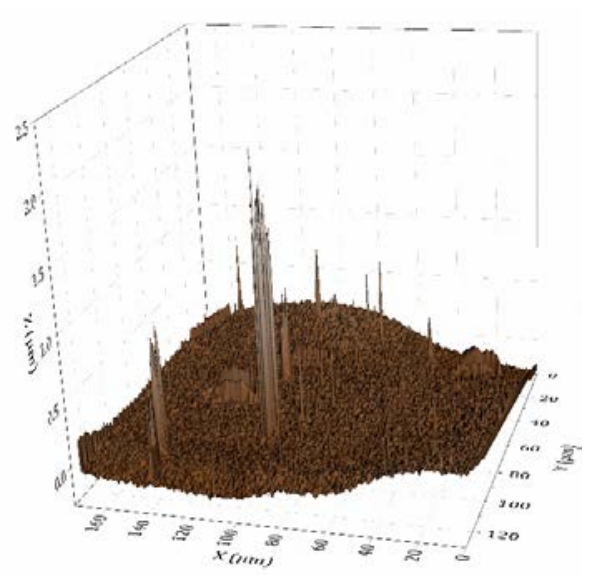

b) PEN/AlOx overview

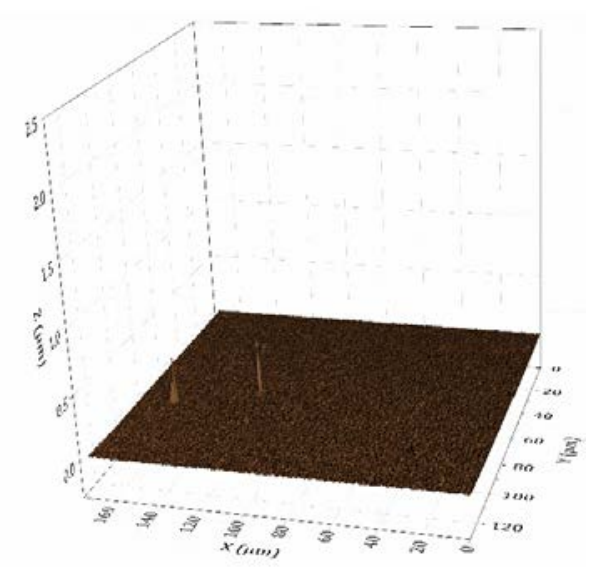

Figure 4: Optical profilometry images of a) an OPP/AIOx surface, and b) a PEN/AIOx. For direct comparison, the images are plotted with a z-scale of $2.5 \mu \mathrm{m}$, over an area $170 \mu \mathrm{m}$ by $130 \mu \mathrm{m}$.

If we examine the topography of the oxide surface in an area of the film away from these larger asperities, we can see, Figure 5, that the oxide surface is still much smoother in the case of the PEN coated film, perhaps giving rise to a lower defect density, including that of nanodefects, in the PEN coated film.

a) OPP/AlOx between the largest asperities

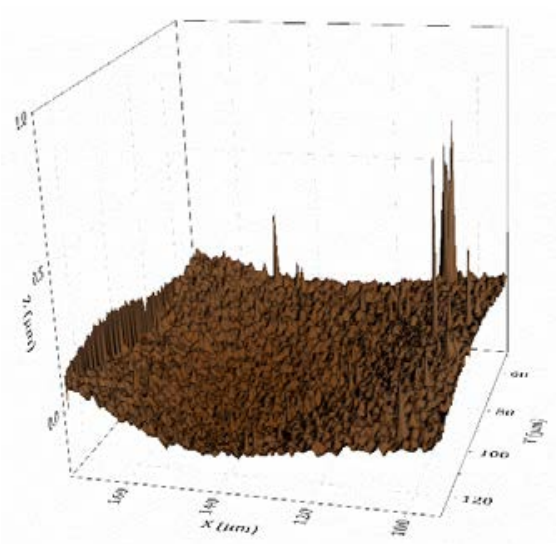

b) PEN/AIOx between the largest asperities

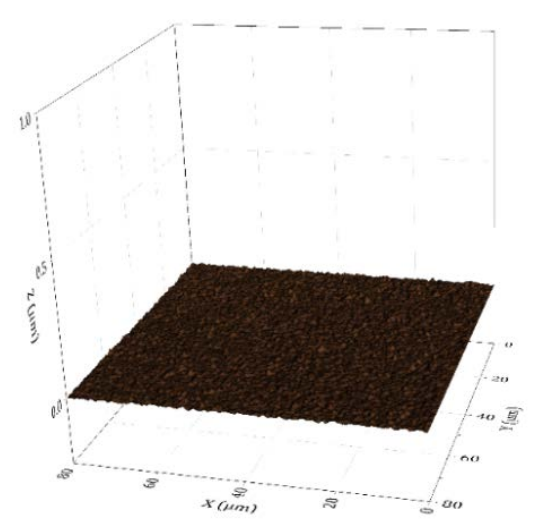

Figure 5: Optical profilometry images of a) an OPP/AIOx surface taken from an area of the surface without asperities over $1 \mu \mathrm{m}$ in height, and b) a PEN/AIOx surface taken from an area without the larger surface asperities. For direct comparison, the images are plotted with a z-scale of $1.0 \mu \mathrm{m}$ over an area of $80 \mu \mathrm{m}$ by 80 $\mu m$.

Such a hypothesis is bourne out when parameters are fitted to equation (2), as reported in Table 1 . In this case the nature of the oxide is assumed to be sufficiently similar that the activation energy of the nanodefects is the same in both cases, and for this, illustrative, data it is arbitrarily set at $130 \mathrm{~kJ} / \mathrm{mol}$. The activation energy of the macrodefects is that measured from the uncoated polymer in each case. Although the absolute figures given in 
Table 1 are not verified, the parameter fits to equation 1, based on the experimental data for composite WVTR, serve to illustrate how the activation energy of the OPP/AIOx composite is greater than that of the PEN/AIOx despite also having a higher level of permeation, as the proportion of permeation that takes place through the nanodefect population is greater in the case of the OPP/AIOx. This correlates with the topography we observe: the overall density of both the macrodefects and the nanodefects in the oxide layer on the rougher OPP is greater and the amount of water vapout passing through both populations is greater in the OPP, but the low level roughness between the large asperities in the oxide my well be indicative of a much greater nanodefect density in the OPP/AIOx case, which results in the greater activation energy.

\section{Conclusions}

The relative contribution to the overall water vapour transmission rate of the macrodefects and nanodefects in a gas barrier coating can be examined by measurement of the apparent activation energy of the composite film. Over the typically small range of temperatures that can be accessed experimentally, the WVTR can be approximated to a single Arrhenius function, and the resulting activation energy will be a reflection of the ratio of the WVTR through the macrodefects and through the nanodefects. Thus the activation energy of a composite completely dominated by macrodefects will be that of the underlying polymer, but will rise as the $W V T R_{\text {nano: }}: W V T R_{\text {macro }}$ increases. This can arise by simply reducing the number of macrodefects, in which case the activation energy will rise and the overall WVTR will decrease, however in the case where the nanodefect density is greater, such as in the example of the OPP/AIOx film, the activation energy can rise despite an increased WVTR. Any change in the activation energy of the nanodefect permeation, such as by changes in the chemistry of the barrier coating, would similarly be expected to change the WVTR and activation energy of the overall composite.

\section{Acknowledgments}

This work was funded by EPSRC studentships with financial support, and provision of substrates, from DuPont Teijin Films and from Innovia Films. The Lucy Halsall Fund, Linacre College is acknowledged for a travel grant.

\section{References}

[1] A.P. Roberts, B.M. Henry, A.P.Sutton, C.R.M. Grovenor, G.A.D.Briggs, T. Miyamoto, M. Kano, Y. Tsukahara, and M. Yanaka, "Gas permeation in silicon-oxide/polymer ( $\left.\mathrm{SiO}_{x} / \mathrm{PET}\right)$ barrier films: role of the oxide lattice, nano-defects and macro defects," J. Membr. Sci. 208 75, 2002 DOI: http://dx.doi.org/10.1016/S03767388(02)00178-3.

[2] C.S. Deng, H.E. Assender, F. Dinelli, O.V.Kolosov, G.A.D.Briggs, T. Miyamoto, and Y. Tskuahara, "Nucleation and growth of gas barrier aluminium oxide on surfaces of poly(ethylene terephthalate) and polypropylene: Effects of the polymer surface properties" J. Polym. Sci. Part B-Polymer Physics 38, 3151, 2000 DOI: http://dx.doi.org/10.1002/1099-0488(20001201)38:23\%3C3151::AID-POLB150\%3E3.0.CO;2-Q.

[3] A.G. Erlat, B.M. Henry, J.J. Ingram, D.B. Mountain, A. McGuigan, R.P. Howson, C.R.M. Grovenor, G.A.D. Briggs, T. Miyamoto, and Y. Tsukahara "Characterisation of aluminium oxynitride gas barrier films" Thin Solid Films 388, 78, 2001 DOI: http://dx.doi.org/10.1016/S0040-6090(01)00836-7.

[4] B. Henry, A.G. Erlat, A. McGuigan, C.R. Grovenor, G.A.D. Briggs, Y. Tsukahara, T.Miyamoto, N. Noguchi, and T. Niijima "Characterization of transparent aluminium oxide and indium tin oxide layers on polymer substrates" Thin Solid Films 382, 194, 2001 DOI: http://dx.doi.org/10.1016/S0040-6090(00)01769-7. 
[5] O.L. Anderson and D.A. Stuart "Calculation of activation energy of ionic conductivity in silica glasses by classical methods" J. Am. Ceram. Soc. 37, 573, 1954 DOI: http://dx.doi.org/10.1111/j.11512916.1954.tb13991.x.

[6] A.G. Erlat, B. Henry, C. Grovenor, A. Briggs, R. Charter, and Y. Tsukahara, "Mechanism of water vapour transport through PET/AlOxNy Gas barrier films" J. Phys Chem B 108, 883, 2004 DOI: http://dx.doi.org/10.1021/jp036244y.

[7] J. Fahlteich, M. Fahland, W. Schönberger, and N. Schiller "Permeation barrier properties of thin films on flexible polymer substrates" Thin Solid Films 517, 3075, 2009 DOI: http://dx.doi.org/10.1016/j.tsf.2008.11.089.

[8] B.M. Henry, A.G. Erlat, C.R.M. Grovenor, C.-S. Deng, G.A.D. Briggs, T. Miyamoto, N. Noguchi, T. Niijima, and Y. Tsukahara, "The permeation of water vapor through gas barrier films", $44^{\text {th }}$ Annual Technical Conference Proceedings, Society of Vacuum Coaters 44, 469, 2001.

[9] A.G. Erlat, R.J. Spontak, R.P. Clarke, T.C. Robinson, P.D. Haaland, Y. Tropsha, N.G. Harvey, and E. A. Vogler, "SiO xas barrier coatings on polymer substrates: morphology and gas transport considerations", J. Phys Chem B 103, 6047, 1999 DOI: http://dx.doi.org/10.1021/jp990737e.

[10] E. Monticone, A.M. Rossi, M. Rajteri, R.S. Gonnelli, V. Lacquaniti, and G. Amato, "Structural and Morphological properties of evaporated SiOx films", Philosophical Magazine Part B 80, 523, 2000 DOI: http://dx.doi.org/10.1080/13642810008209760.

[11] Y. Leterrier, J. Andersons, Y. Pitton and J.E. Manson, "Adhesion of silicon oxide layers on poly(ethylene terephthalate. II: Effect of coating thickness on adhesive and cohesive strengths, J. Polym Sci. Part B 35, 1463, 1997 DOI: http://dx.doi.org/10.1002/(SICI)1099-0488(19970715)35:9\%3C1463::AIDPOLB16\%3E3.0.CO;2-4.

[12] B.M. Henry, H.E. Assender, A.G. Erlat, C.R.M Grovenor, G.A.D. Briggs, T. Miyamoto, and Y. Tsukahara, "Gas barrier properties of transparent metal oxide coatings on PET film" $47^{\text {th }}$ Annual Technical Proceedings, Society of Vacuum Coaters 47, 609, 2004.

[13] G. Rossi, and M. Nulman, "Effect of local flaws in polymeric permeation reducing barriers" J. Appl. Phys. 74, 5471, 1993 DOI: http://dx.doi.org/10.1063/1.354227. 